\title{
Midbrain-Driven Emotion and Reward Processing in Alcoholism
}

\author{
EM Müller-Oehring*, ',2, Y-C Jung ${ }^{2,3}$, EV Sullivan' ${ }^{2}$, WC Hawkes ${ }^{1,2}$, A Pfefferbaum ${ }^{1,2}$ and T Schulte' \\ 'Neuroscience Program, Center Health Science, SRI International, Menlo Park, CA, USA; ${ }^{2}$ Department of Psychiatry and Behavioral Sciences, \\ Stanford University, Stanford, CA, USA; ${ }^{3}$ Department of Psychiatry, Yonsei University, College of Medicine, Seoul, South Korea
}

\begin{abstract}
Alcohol dependence is associated with impaired control over emotionally motivated actions, possibly associated with abnormalities in the frontoparietal executive control network and midbrain nodes of the reward network associated with automatic attention. To identify differences in the neural response to alcohol-related word stimuli, 26 chronic alcoholics (ALC) and 26 healthy controls (CTL) performed an alcohol-emotion Stroop Match-to-Sample task during functional MR imaging. Stroop contrasts were modeled for color-word incongruency (eg, word RED printed in green) and for alcohol (eg, BEER), positive (eg, HAPPY) and negative (eg, MAD) emotional word content relative to congruent word conditions (eg, word RED printed in red). During color-Stroop processing, ALC and CTL showed similar left dorsolateral prefrontal activation, and CTL, but not ALC, deactivated posterior cingulate cortex/cuneus. An interaction revealed a dissociation between alcohol-word and color-word Stroop processing: ALC activated midbrain and parahippocampal regions more than CTL when processing alcohol-word relative to color-word conditions. In ALC, the midbrain region was also invoked by negative emotional Stroop words thereby showing significant overlap of this midbrain activation for alcohol-related and negative emotional processing. Enhanced midbrain activation to alcohol-related words suggests neuroadaptation of dopaminergic midbrain systems. We speculate that such tuning is normally associated with behavioral conditioning to optimize responses but here contributed to automatic bias to alcohol-related stimuli.

Neuropsychopharmacology (2013) 38, I844-1853; doi:I0.1038/npp.2013.102; published online 29 May 2013
\end{abstract}

Keywords: functional connectivity; functional MRI; automatic attentional bias; alcohol; emotion; Stroop task; Alcohol Use Disorder

\section{INTRODUCTION}

Deficits in processing emotion and in controlling impulsivity are frequently observed in alcoholics (ALC) (Clark et al, 2007; Foisy et al, 2007, Maurage et al, 2011; Montagne et al, 2006; Oscar-Berman et al, 1990; Salloum et al, 2007; Townshend and Duka, 2003), suggesting that specific abnormalities in the structure and function of neural networks that regulate emotion are compromised (Phillips et al, 2008; Schulte et al, 2010). Emotion and reward systems closely interact (Morrison and Salzman, 2009). In ALC, for example, anxiety ratings correlated with parahippocampal activation to emotionally negative images, but this association was absent when negative emotion and alcohol stimuli were presented together suggesting that alcohol cues can modulate cortical networks and decrease responsiveness to negative emotion (Gilman and Hommer, 2008).

Neuroanatomically, the reinforcing and rewarding alcohol effects are transmitted via dopaminergic and

\footnotetext{
* Correspondence: Dr EM Müller-Oehring, Department of Psychiatry and Behavioral Sciences, Stanford University School of Medicine, 401 Quarry Road, Stanford, CA 94305, USA, Tel: + I 6508592767, Fax: + 650 8592743, E-mail: evamoe@stanford.edu Received 2 January 2013; revised 18 March 2013; accepted 3 April 2013; accepted article preview online 24 April 2013
}

glutamatergic projections in the mesolimbic corticostriatal system, which originates in midbrain nodes and connects to the limbic system via the nucleus accumbens, amygdala, and hippocampus, and to the medial prefrontal cortex (Berridge and Robinson, 2003; Di Chiara, 2002). Several brain areas associated with emotional dysfunction are affected in ALC (Gainotti, 2001; Oscar-Berman and Bowirrat, 2005; Sinha and $\mathrm{Li}, 2007$ ), including the limbic system (Beresford et al, 2006; Glahn et al, 2007; Grüsser et al, 2004; Schneider et al, 2001), frontal cortices (Mann et al, 2001; Oscar-Berman, 2000; Pfefferbaum et al, 1997; Sullivan et al, 2000), cerebellum (Phillips et al, 1987; Schneider et al, 2001; Sullivan et al, 2000, 2003), and dopamine-rich basal ganglia (De Rosa et al, 2004; Sullivan et al, 2005). Alcohol abuse further affects components of selective attention and executive control (Evert and OscarBerman, 2001; Müller-Oehring et al, 2009; Schulte et al, 2005 ; 2012), mediated predominantly by cortical networks involving parietal (Fein and Chang, 2006; Schulte et al, 2005) and frontal association areas and the anterior cingulum (Bush et al, 2000; Devinsky et al, 1995; Le Berre et al, 2012).

Recent research is focusing on the disruption of neural connectivity of the brain circuits that regulate executive control in alcoholism (Schulte et al, 2012) and interact with emotion processing (O'Daly et al, 2012). Selective brain 
systems involving corticostriatal brain pathways have a crucial role in a tendency to experience negative emotion in alcoholism (Makris et al, 2008; Wrase et al, 2008; Marinkovic et al, 2009; Oberlin et al, 2012; Schulte et al, 2010; Vollstädt-Klein et al, 2012). In a functional neuoimaging study, we elucidated abnormalities in the frontoparietal attention and executive control network in alcohol dependence, and in midbrain nodes of the reward network associated with automatic attention functions (Schulte et al, 2012). Although it can be assumed that words connoting alcohol gain emotional relevance with continued alcohol abuse and can automatically capture attention (Field et al, 2012), it remains elusive whether such implicit attentional bias to alcohol-related stimuli is processed in different or overlapping neural networks relative to emotional stimuli engaging the limbic system (Fitzgerald et al, 2006; Gilman et al, 2008).

To further understanding about the interplay between frontoparietal executive control and midbrain-based reward networks in chronic alcoholism for the regulation of alcohol- and emotion-related interference, we developed an alcohol-emotion Stroop Match-to-Sample task for use in functional magnetic resonance imaging (fMRI) paradigms. Our task, which requires subjects to match the color of a cue to that of a word, recently showed that patients with alcoholism have greater difficulty than controls (CTL) in resolving conflict arising from emotional words (Schulte et al, 2011) similar to the observed attentional bias to alcohol words (Field et al, 2012; Johnsen et al, 1994). The overarching hypothesis of the current study is that chronic alcohol consumption compromises neural regulation of prefrontal monitoring and midbrain reward mechanisms within the mesocorticolimbic system. This compromise may lead to a better understanding of the neural substrates of alcohol cue-related behavior and relapse in abstinent ALC. Specifically, we tested the hypotheses that in ALC, alcohol words would automatically capture attention and induce interference as do emotional words, and that reward-related mesocorticolimbic midbrain regions would modulate frontoparietal attention and executive control systems in ALC differently from that in CTL while processing interference from alcohol and emotion words in a Stroop task.

\section{MATERIALS AND METHODS}

\section{Participants}

Groups were comprised of 26 abstinent alcoholic men and 26 age-matched control men and women. ALC were recruited from local rehabilitation programs; CTL were volunteers from the local community. All subjects were screened with the Structural Clinical Interview for DSM-IV (American Psychiatric Association, 2000) and a clinical examination to rule out other Axis I diagnoses or non-alcohol substance abuse. In the alcoholic group, 25 participants met DSM-IV criteria for alcohol dependence and 1 participant met criteria for alcohol abuse. Of those meeting dependence criteria, 24 were in early remission (met criteria within the past 12 months), whereas 1 was in sustained remission ( $>12$ months). The median number of weeks since ALC last met dependence criteria was 17 weeks
( mean $=16.0$ weeks, SD $=12.8$ weeks). The median age of alcoholism onset was 25 years $($ mean $=29.1, \mathrm{SD}=13.6)$. In all, $46 \%$ of ALC and $0 \%$ of CTL met DSM-IV dependence for any type of drug dependence. The most common type of drug dependence among ALC was cocaine (endorsed by $31 \%$ of ALC), and the median number of year since last used cocaine was 1.3 years (mean $=9.5$ years, $S D=10$ years, range $=0.5-11.5$ years). Other types of drug dependency were amphetamines in $12 \%$ and opiods in $12 \%$ of ALC. The median number of years since last used amphetamines was 6.5 years $($ mean $=6$ years, $S D=5.5$ years, range $=0.5-24.5$ years), and for opiods 2 years (mean $=10$ years, $S D=14.75$ years, range $=1.2-27$ years). In no case was drug dependence more recent than alcohol dependence. Significantly, more ALC met DSM-IV criteria for nicotine dependence (54\% current smokers, $23 \%$ past smokers) than did CTL ( $12 \%$ current smokers), $\chi^{2}(2)=23.08, P=0.0001$ (Fisher's exact test). Subject groups matched in age and handedness (Table 1), and did not differ in visual acuity $(t=1.01$, $P=0.32$ ), body mass index (CTL: $27 \pm 4$; ALC: $27 \pm 6$; $t=0.00, P=0.99)$, and physiological measures such as heart rate (CTL: $72 \pm 12$; ALC: $77 \pm 11 ; t=-1.59, P=0.12$ ) and blood pressure (systolic CTL: $126 \pm 14$; ALC: $128 \pm 18$; $t=-0.39, P=0.7$; diastolic CTL: $73 \pm 7$; ALC: $76 \pm 12$; $t=-1.33, P=0.19)$.

Groups differed in questionnaire-based mood characteristics; compared with CTL, ALC had higher depressive symptoms (BDI-II, Beck et al, 1996; $t=4.99, P<0.0001$ ), trait anxiety (STAI-T; Spielberger et al, 1983; $t=4.19$, $P<0.0001$ ), and state anxiety scores (STAI-S; $t=2.27$, $P=0.028$; Table 1$)$. Although both groups had an average education beyond high school level, CTL had significantly more years of education than ALC (Table 1).

All participants gave written informed consent to participate in this study, which was approved by the Institutional Review Boards at SRI International and Stanford University School of Medicine.

\section{Stimuli and Experimental Design}

Study participants underwent cognitive testing and additional behavioral testing during fMRI image acquisition. Structural and functional MR imaging data were acquired using a clinical whole-body GE 3T scanner. Using a backprojection system during the fMRI session, participants viewed stimuli on a mirror attached to the head coil.

Alcohol and emotion Stroop match-to-sample task. Stimuli were created and presented with PsyScope software (Cohen et al, 1993) and were synchronized with magnetic resonance imaging (MRI) acquisition via the fORB system interface (http://www.curdes.com). Subjects matched the color of a cue stimulus displayed for $700 \mathrm{~ms}$ in the center of the screen to the color of a Stroop target stimulus that appeared for $700 \mathrm{~ms}$ after a color mask, consisting of a gray patch, that was presented for $700 \mathrm{~ms}$ between cue and target. Cue and target colors were red, green, or blue. Total trial duration was $4.4 \mathrm{~s}$ including inter-stimulus intervals of 300-700 ms between color cue, mask, and Stroop target. The color cue either matched or did not match the color of the Stroop target, which was either congruent (word BLUE written in blue font color) or incongruent (word BLUE 
written in red font color). Incongruent Stroop words were color words (RED, GREEN, BLUE), alcohol words (eg, WINE, BEER, WHISKEY), and emotionally positive (eg, HAPPY, JOY) and negative words (eg, MAD, ANGRY) printed in green, red, or blue front color (Figure 1). The Stroop effect is defined as the difference in reaction time (RT) to incongruent and congruent stimuli. For incongruent-nonmatch color-Stroop conditions, the cue color always matched the word content (eg, red cue, word RED written in green font color) (Schulte et al, 2005, 2008, 2009, 2011, 2012).

Subjects pressed a YES key for cue-target color matches and a NO key for nonmatches using their dominant hand, yielding accuracy and RT measures. To test the effect of repetitive behavior on cognitive control and conflict resolution, we presented trials in two block types: mixed and same response blocks (Figure 1). In mixed response blocks, both match and nonmatch trials were presented and required switching between YES and NO responses, whereas same response blocks contained either match (YES responses) or nonmatch trials (NO responses) and did not require response switches. To quantify Stroop effects within a block design, incongruent and congruent trials were never mixed within a block. Mixed response and same response blocks comprised the same number of trials. Total number of trials was 60 per run. Two runs were presented with 15 blocks each (block $=8$ TRs; $\quad \mathrm{TR}=2.2 \mathrm{~s}$ ). Test instructions were reviewed with the subject in a practice session before entering the scanner and again through the scanner's intercom system before each run. Subjects had a short break between run 1 and run 2, but remained in the scanner.

\section{MRI}

Data acquisition and analyses. Whole-brain structural and functional MRI data were acquired with an 8-channel head coil at a $3 \mathrm{~T}$ GE whole-body scanner. Subject motion was minimized by following best practices for head fixation, and structural image series were inspected for residual motion. Whole-brain fMRI data were acquired with a $\mathrm{T} 2{ }^{*}$-weighted gradient echo planar pulse sequence (2D axial, $\mathrm{TE}=30 \mathrm{~ms} ; \mathrm{TR}=2200 \mathrm{~ms}$; flip angle $=90^{\circ}$; in plane resolution $=3.75 \mathrm{~mm}$; thick $=5 \mathrm{~mm}$; skip $=0 \mathrm{~mm}$; locations $=36 ; \mathrm{FOV}=240 \mathrm{~mm} ; 1 \mathrm{NEX}$ ). The start of the scan was triggered automatically from PsyScope software. Test instructions were reviewed with the subject by the examiner via the scanner intercom system before the onset of each run. A dual-echo FSE (2D axial; TR $=5000 \mathrm{~ms}$; $\mathrm{TE}=17 / 102 \mathrm{~ms} ; \quad$ thick $=5 \mathrm{~mm} ; \quad$ skip $=0 \mathrm{~mm} \mathrm{~mm} ; \quad x y$ matrix $=256$; flip angle $=90^{\circ}$; locations $=36 ; \mathrm{FOV}=240$ $\mathrm{mm}$; $1 \mathrm{NEX)}$ was used for spatially registering the fMRI data. A field map for correction of spatial distortions in the echo-planar images was generated from a gradientrecalled echo sequence pair $(\mathrm{TR}=460 \mathrm{~ms}, \mathrm{TE}=3 / 5 \mathrm{~ms}$, thickness $=5 \mathrm{~mm}$, skip $=0 \mathrm{~mm}$, locations $=36$ ).

Image preprocessing and statistical analyses were performed using the SPM8 software package (Wellcome Department of Cognitive Neurology). The fMRI analysis focused on the whole brain. The functional images were subjected to geometric distortion (field map) correction and motion correction. The FSE structural images were 
Alcohol- and Emotion-Stroop Match-to-Sample Paradigm

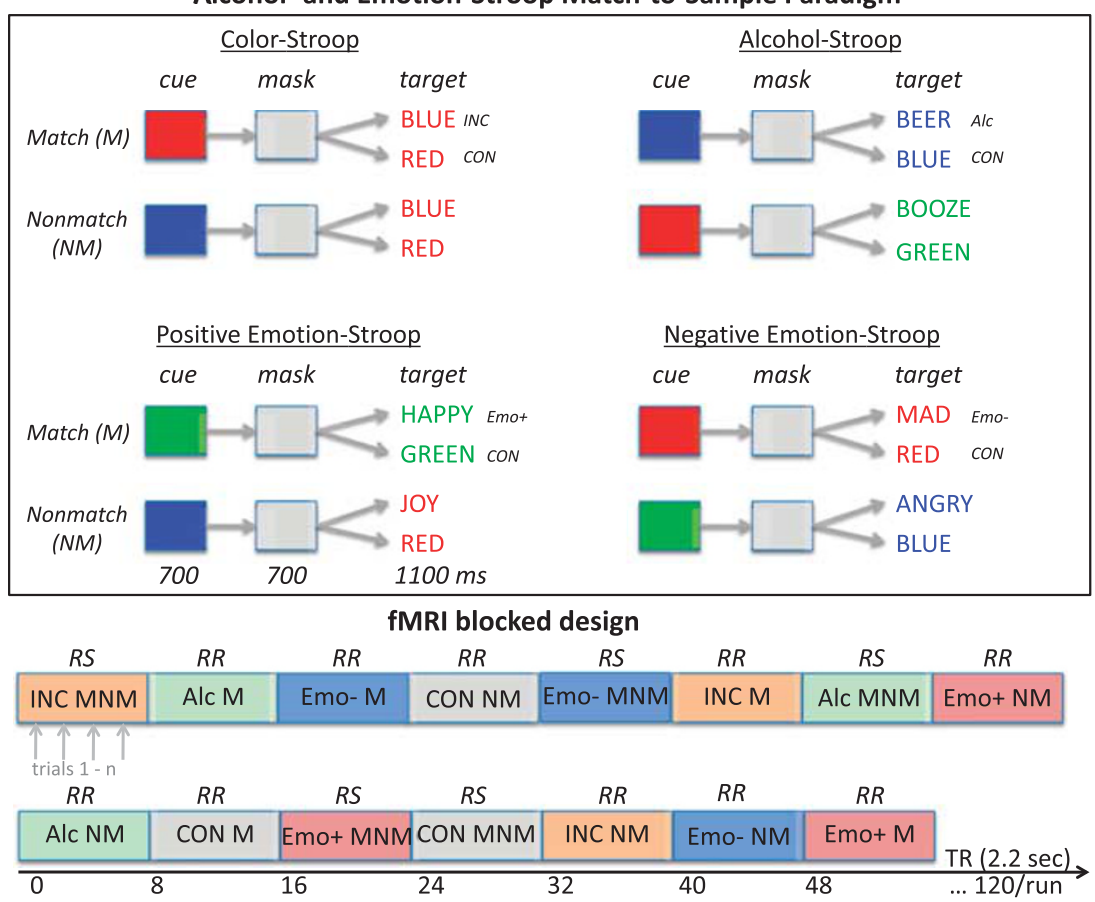

Figure I Alcohol and emotion Stroop match-to-sample paradigm (upper panel): the task was to match the color of a color cue to that of a target word by pressing a YES key for color matches and a NO key for color nonmatches. The task had four Stroop content conditions: (I) incongruent color word (INC), (2) alcohol words (Alc), (3) positive emotion words (Emo +), and (4) negative emotion words (Emo - ). Incongruent Stroop words were color words (RED, GREEN, BLUE), alcohol words (eg, WINE, BEER, WHISKEY), emotionally positive (eg, HAPPY, JOY), and negative words (eg, MAD, ANGRY) printed in green, red, or blue front color. The Stroop effect is defined as the difference in reaction time (RT) to incongruent and congruent stimuli (CON), and was computed for all four conditions, for color matches (M) and nonmatches (NM). Trials were presented in two block types (lower panel): In response switching blocks (RS), both match and nonmatch trials were presented (MNM) and required switching between YES and NO responses; response repetition blocks (RR) did not require response switches.

co-registered to the mean unwarped and motion-corrected functional image for each subject and segmented into gray and white matter images. Functional and structural gray matter images were normalized to Montreal Neurological Institute space, and volumes were smoothed with a Gaussian kernel of $8 \mathrm{~mm}$ (FWHM). Individual statistics were computed using a general linear model approach (Friston et al, 1995) as implemented in SPM8. Statistical preprocessing consisted of high-pass filtering at $88 \mathrm{~s}$, lowpass filtering through convolution with the canonical hemodynamic response function, and global scaling. A random effects analysis was conducted for group averaging and population inference. One image per contrast was computed for each subject from a design matrix that included estimated individual movement parameters as regressors in addition to Stroop task conditions as explanatory variables. Stroop contrasts of interest were Color Stroop derived by contrasting 'INC $>$ CON' conditions, Alcohol Stroop derived by contrasting 'Alc $>$ CON' conditions, Postitive-Emotion Stroop derived by contrasting 'Emo $+>$ CON' conditions, and Negative-Emotion Stroop derived by contrasting 'Emo- $>$ CON' conditions computed for response repetition and response switching conditions (see Figure 1). For second-level group analyses, these Stroop contrast images were subjected to a factorial model involving two factors: Group (ALC vs CTL) and Stroop (color, alcohol, positive-emotion, negative-emotion). The second-level factorial model allows analysis of group and condition main effects and interactions, and conjunction analyses to identify areas of spatial overlap between the statistical maps from the two groups (Friston et al, 2005; Nichols et al, 2005). For functional connectivity analysis, the 'conn' toolbox was used as implemented SPM8. Seed-to-voxel connectivity maps for each group were derived via individual time series correlations of activity over 244 time points, an index of synchronous activity. Analyses thresholds were set at $P_{\mathrm{FDR}}$ corrected $<0.05$ for combined spatial extent and peak intensity (Poline et al, 1997).

\section{Statistical Analyses}

For behavioral data analysis, repeated-measures analysis of variance tested for group effects (ALC, CTL) as betweensubjects factor and to test for Stroop effects (incongruent/ congruent), color match effects (nonmatch/match), and response block effects (mix/same) as within-subject factors. To examine the relationships predicted between task-related functional regional brain activation and behavioral measures of Stroop performance, we used two-tailed Pearson correlations in each group separately. One-tailed Pearson correlation tested directional hypotheses on the effects of lifetime alcohol consumption on behavior and brain function. The alpha level was set to 0.05 for all statistical tests, including hypotheses-driven analyses of structurefunction relationships. FDR corrected $P$ levels were applied for multiple comparisons where applicable (Benjamini et al, 2001). 


\section{RESULTS}

\section{Behavioral Results: Stroop Match-to-Sample Task}

$R T$ and accuracy. Groups did not differ in processing speed (RTs; ALC $781 \pm 149 \mathrm{~ms} ; \quad$ CTL $757 \pm 134 \mathrm{~ms}$; $t(50)=0.61, n s$ ) or accuracy (number of errors; ALC $3 \pm 2.4$; CTL $3 \pm 2.1 ; t(50)=0.19, n s)$. With an average error rate of $2.5 \%$ ( 3 out of 120 trials), the number of errors was low in each group precluding further error analysis. In neither group were speed and accuracy correlated significantly (ALC $r=-0.06$, ns; CTL $r=0.25$, ns), which reflects the absence of a speed-accuracy trade-off.

Stroop effects. Analysis of variance for each Stroop effect with color cueing (match, nonmatch) and response block (repetition, switching) as within-subject factors and group (ALC, CTL) as between-subjects factor were conducted. Overall, Stroop effects did not differ between groups (Table 2). A trend for greater color Stroop effects (INC, CON) was observed in ALC than CTL, specifically for color Stroop during response repetition.

\section{Group Differences in Stroop Task-related Brain Activity}

Significant group-by-condition interactions were observed for alcohol Stroop and for positive-emotion Stroop (Table 3C), demonstrating that (a) ALC activated midbrain and parahippocampal brain regions more than CTL when processing alcohol Stroop (Alc vs CON) relative to color Stroop (INC vs CON) (Figure 2a), and (b) ALC activated dorsolateral prefrontal cortex (DLPFC) regions less than CTL when processing positive-emotion Stroop $(E m o+v s$ CON) relative to color Stroop (INC vs CON; Figure 2a). In ALC, low DLPFC activation was further modulated by current smoking: smoking status (no, past, current smoker) affected DLPFC activity during positive-emotion processing (ANCOVA, $F(223)=3.81, P=0.037$ ). Here, post-hoc LSD analysis showed significantly lower DLPFC activity in current smokers than non-smokers $(P=0.02)$, and a trend for lower DLPFC activity in current compared with past smokers $(P=0.076)$, whereas non-smokers and past

Table 2 Stroop Means and SDs for Each Group: ALC and CTL; for Color, Alcohol, Negative- and Positive-Emotion Stroop,

Differentiated by Task Modulation: Cue Color Matches and Response blocks

\begin{tabular}{llllll}
\hline Stroop effect & & ALC & CTL & F & P \\
\hline Color & Total & $60.8 \pm 94$ & $22.2 \pm 59$ & 3.03 & 0.088 \\
Cue color & Match & $68.9 \pm 123$ & $33.5 \pm 72$ & 1.56 & NS \\
& Nonmatch & $52.7 \pm 130$ & $11.0 \pm 83$ & 1.84 & \\
Response block & Repetition & $78.7 \pm 133$ & $13.9 \pm 74$ & 4.59 & 0.037 \\
& Switching & $42.9 \pm 105$ & $30.6 \pm 93$ & 0.19 & NS \\
Alcohol & Total & $46.3 \pm 104$ & $15.8 \pm 59$ & 1.66 & NS \\
Negative Emotion & Total & $-4.2 \pm 111$ & $-18.5 \pm 63$ & 0.01 & NS \\
Positive Emotion & Total & $-14.2 \pm 78$ & $-12.1 \pm 53$ & 0.32 & NS \\
\hline
\end{tabular}

Abbreviations: ALC, alcoholic; CTL, control; NS, non significant.

MANOVA group statistics $(F, P)$.

Significance level at $P<0.05$.

Italic values are $P$. smokers did not differ $(P=0.59)$. Using education or SES as covariate did not significantly change the results.

Follow-up analysis on similarities and differences between study groups for each Stroop condition revealed for color Stroop: an overlap in DLPFC activity, for alcohol Stroop: greater midbrain and parahippocampal activity in ALC than CTL, and for positive-emotion Stroop: greater left DLPFC activity in CTL than ALC (Table 3b).

\section{Stroop Task-Related Brain Activity}

Color Stroop. When processing incongruent relative to congruent Stroop color words, a left frontoparietal network was engaged. CTL activated left medial frontal and occipital brain regions and deactivated medial parietal (posterior cingulate cortex [PCC], cuneus, precuneus) and subcortical regions (thalamus, claustrum). ALC activated left DLPFC and deactivated temporal, premotor, and somatosensory brain regions (Supplementary Table 1).

Alcohol Stroop. When processing alcohol-related relative to congruent Stroop words, midbrain and occipital

Table 3 Brain Activation to Stroop Interference from Color, Alcohol, Negative, and Positive-Emotion words in ALC and CTL

\begin{tabular}{|c|c|c|c|c|c|c|}
\hline \multirow[b]{2}{*}{ Region } & \multirow[b]{2}{*}{ BA } & \multirow[b]{2}{*}{$\begin{array}{l}\text { P FDR } \\
\text { extent }\end{array}$} & \multirow[b]{2}{*}{$T$} & \multicolumn{3}{|c|}{ MNI coordinates } \\
\hline & & & & $x$ & $y$ & $\mathbf{z}$ \\
\hline \multicolumn{7}{|l|}{ (A) Group-by-Stroop interaction ${ }^{a}$} \\
\hline Alcohol Stroop > color Stroop & \multicolumn{3}{|c|}{$A L C>C T L$} & & & \\
\hline L Parahippocampal gyrus & 19 & 0.0001 & 3.75 & -30 & -44 & -10 \\
\hline L Midbrain — substantia nigra & & 4 & & -10 & -24 & -12 \\
\hline R Midbrain—red nucleus & & & & 2 & -28 & -12 \\
\hline Positive-emotion Stroop > color Stroop & \multicolumn{3}{|c|}{$A L C<C T L$} & & & \\
\hline \multirow[t]{3}{*}{ L DLPFC — middle frontal gyrus } & 9 & 0.009 & 3.97 & -44 & 10 & 40 \\
\hline & 6 & & 3.93 & -40 & 6 & 50 \\
\hline & 9 & & 3.69 & -34 & 22 & 26 \\
\hline \multicolumn{7}{|l|}{ (B) Group effects ${ }^{b}$} \\
\hline Color Stroop & \multicolumn{3}{|c|}{$C T L=A L C$} & & & \\
\hline L DLPFC - middle frontal gyrus & 46 & $0.094^{c}$ & 3.69 & -44 & 16 & 22 \\
\hline $\mathrm{L}$ — middle frontal gyrus & 9 & & & -48 & 20 & 38 \\
\hline $\mathrm{L}$-inferior frontal gyrus & 9 & & & -42 & 6 & 24 \\
\hline Alcohol Stroop & \multicolumn{3}{|c|}{$A L C>C T L$} & & & \\
\hline$L+R$ Midbrain & & 0.005 & 3.75 & 0 & -30 & -10 \\
\hline L Parahippocampal gyrus & \multirow{2}{*}{\multicolumn{2}{|c|}{19}} & & -30 & -44 & -8 \\
\hline R Midbrain_-red nucleus & & & & 6 & -20 & -10 \\
\hline Negative-emotion Stroop & \multicolumn{3}{|c|}{ No suprathreshold voxels } & & & \\
\hline Positive-emotion Stroop & \multicolumn{3}{|c|}{$A L C<C T L$} & & & \\
\hline \multirow[t]{3}{*}{ L DLPFC — middle frontal gyrus } & 9 & $0.096^{c}$ & 3.66 & -44 & 10 & 40 \\
\hline & 6 & & 3.37 & -40 & 6 & 50 \\
\hline & 9 & & 3.29 & -34 & 24 & 24 \\
\hline
\end{tabular}

${ }^{a}$ Group-by-Stroop interaction; $P_{\text {FDR-corrected }}<0.05$ for extent for the wholebrain volume and $P_{\text {uncorr }}<0.001$ for peak.

${ }^{b}$ Group effects; $P_{\text {FDR-corrected }}<0.05$ for combined peak and extent threshold (Poline et al, 1997).

'Statistical trend, $P_{\text {FDR-corrected }}>0.05$ and $<0.01$. 

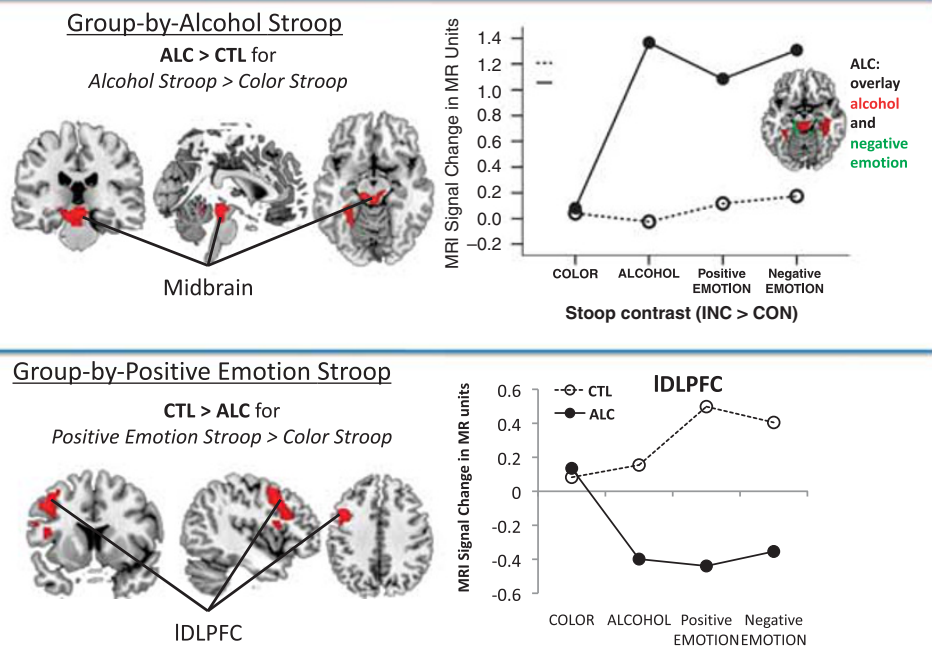

b

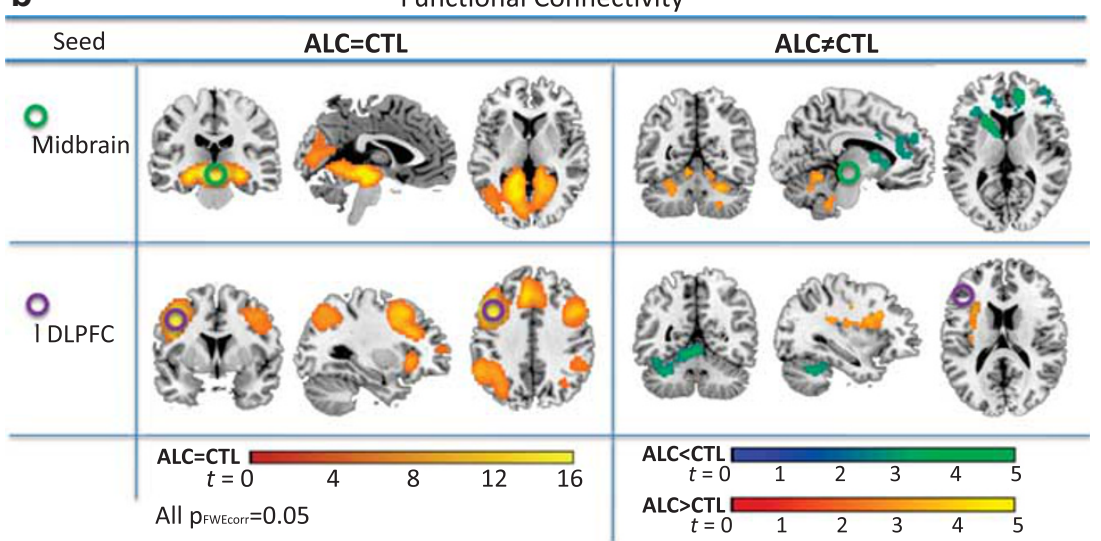

Figure 2 Functional magnetic resonance imaging ( $\mathrm{MRR}$ ): (a) Group-by-Stroop interaction showing that alcoholics (ALC) activated midbrain and parahippocampal brain regions more than CTL when processing alcohol Stroop (Alc > CON) relative to color Stroop (INC > CON), and (lower panel) CTL activated dorsolateral prefrontal cortex (DLPFC) regions more than ALC when processing positive-emotion Stroop (Emo $+>C O N$ ) relative to color Stroop (INC>CON). Graphs illustrating MRI signal change in the midbrain-hippocampal (upper panel) and left DLPFC clusters (lower panel) in ALC and CTL for color Stroop, alcohol Stroop, positive-emotion Stroop, and negative-emotion Stroop. (b) fcMRI: Seed-to-voxel synchronous activation for the midbrain seed (upper panel) and the DLPFC seed (lower panel) for group similarities (left) and group differences (right).

regions were engaged. Although CTL mainly deactivated medial parietal regions (PCC, cuneus, precuenus; see also Table 3a), ALC activated midbrain and parahippocampal regions and deactivated motor and somatosensory regions (pre- and postcentral gyri; Supplementary Table 1).

Negative-emotion Stroop. Processing negative-emotion relative to congruent Stroop words activated a cerebellarmidbrain cluster. In CTL, however, brain activity to negativeemotion words did not differ from that to congruent color words. ALC activated midbrain and parahippocampal gyri, and deactivated pre- and postcentral gyri. A conjunction analysis in ALC comparing 'alcohol Stroop' and 'negative-emotion Stroop' activity revealed significant overlap of activation and deactivation patterns in midbrain, hippocampal, motor, and somatosensory regions (Supplementary Table 1).

Positive-emotion Stroop. When processing positiveemotion words relative to congruent words, CTL activated the left DLPFC. In ALC, processing positive-emotion words did not invoke significantly different activity from processing congruent color words (Supplementary Table 1).

\section{Functional Brain Networks}

To study the functional networks of midbrain and left DLPFC regions showing group-by-Stroop interactions, we used task-related functional connectivity analysis testing synchronous activation patterns between the seed region and voxels in the whole brain (see Table 3, Figure 2).

Midbrain seed. Compared with CTL, ALC showed significantly less midbrain-frontal connectivity and more midbrain connectivity to thalamic and cerebellar regions (Table 4, Figure 2b). Functional midbrain connectivity to left lingual and right parahippocampal and middle temporal gyri overlapped in ALC and CTL. 
Table 4 Functional Connectivity Analysis for Midbrain and IDLPFC Seed Regions in ALCs and CTLs

\begin{tabular}{|c|c|c|c|c|c|c|}
\hline \multirow[b]{2}{*}{ Region } & \multirow[b]{2}{*}{ BA } & \multirow{2}{*}{$\begin{array}{c}\text { Extent } \\
k \# \text { voxels }\end{array}$} & \multirow[b]{2}{*}{$T$} & \multicolumn{3}{|c|}{ MNI coordinates } \\
\hline & & & & $x$ & $y$ & $z$ \\
\hline \multicolumn{7}{|l|}{ Midbrain functional connectivity } \\
\hline \multicolumn{7}{|l|}{$A L C<C T L$} \\
\hline L Putamen & & 3311 & 4.98 & -22 & 16 & 8 \\
\hline R mPFC & 10 & & 4.51 & 16 & 42 & 10 \\
\hline R DLPFC—-superior frontal gyrus & 9 & & 4.12 & 38 & 40 & 34 \\
\hline R DLPFC — superior frontal gyrus & 10 & 701 & 3.51 & 30 & 56 & 18 \\
\hline$R$ —superior frontal gyrus & 9 & & 3.27 & 8 & 56 & 26 \\
\hline R—middle frontal gyrus & 10 & & 3.23 & 26 & 60 & 26 \\
\hline \multicolumn{7}{|l|}{$A L C>C T L$} \\
\hline L Caudate tail & & 841 & 4.84 & -32 & -26 & -6 \\
\hline L Thalamus & & & 4.30 & -26 & -34 & 0 \\
\hline \multirow[t]{3}{*}{ R Anterior cerebellum—4, 5} & & 885 & 4.25 & 26 & -54 & -30 \\
\hline & & & 3.60 & 28 & -46 & -34 \\
\hline & & & 3.47 & 20 & -38 & -34 \\
\hline \multirow[t]{2}{*}{ L Anterior cerebellum— 4,5} & & 898 & 3.66 & -10 & -54 & -12 \\
\hline & & & 3.45 & -24 & -52 & -32 \\
\hline \multicolumn{7}{|l|}{$A L C=C T L$} \\
\hline L Lingual gyrus & 30 & 21906 & 32.35 & -16 & -40 & -4 \\
\hline R Parahippocampal gyrus & 30 & & 29.95 & 20 & -38 & -8 \\
\hline L Lingual gurus & 19 & & 28.80 & -16 & -52 & -2 \\
\hline \multirow[t]{2}{*}{ R Middle temporal gyrus } & 19 & 288 & 6.87 & 34 & -84 & 22 \\
\hline & 19 & & 6.46 & 42 & -82 & 18 \\
\hline R Angular gyrus & 39 & & 6.13 & 36 & -80 & 30 \\
\hline \multicolumn{7}{|l|}{ IDLPFC functional connectivity } \\
\hline \multicolumn{7}{|l|}{$A L C<C T L$} \\
\hline L Anterior cerebellum—4, 5 & & 1801 & 4.11 & 0 & -52 & -8 \\
\hline R Cerebellum—crus I & & & 4.05 & 30 & -70 & -26 \\
\hline L Anterior cerebellum—4, 5 & & & 4.01 & -8 & -54 & -14 \\
\hline $\mathrm{L}$ medial prefrontal cortex, $\mathrm{ACC}$ & 10 & 900 & 3.81 & -8 & 38 & -4 \\
\hline L Inferior frontal gyrus & 11 & & 3.79 & -10 & 40 & -22 \\
\hline R ACC & 32 & & 3.61 & 6 & 38 & -8 \\
\hline \multicolumn{7}{|l|}{$A L C>C T L$} \\
\hline L Insula & 13 & 1228 & 4.20 & -30 & 10 & 24 \\
\hline $\begin{array}{l}\text { L Middle frontal and medial } \\
\text { temporal gyri }\end{array}$ & 9,48 & & 4.13 & -32 & 18 & 30 \\
\hline \multicolumn{7}{|l|}{$A L C=C T L$} \\
\hline \multirow[t]{2}{*}{ L DLPFC—-middle frontal gyrus } & 9 & 12902 & 33.55 & -44 & 12 & 34 \\
\hline & 9 & & 15.27 & -6 & 30 & 40 \\
\hline L Middle cingulate cortex, SMA & 32 & & 14.27 & -8 & 24 & 46 \\
\hline \multirow[t]{2}{*}{ L Inferior parietal lobe } & 40 & 4027 & 16.68 & -52 & -44 & 46 \\
\hline & 39 & & 12.53 & -44 & -56 & 48 \\
\hline L Superior parietal lobe & 7 & & 11.88 & -36 & -64 & 52 \\
\hline $\mathrm{R}$ Inferior frontal gyrus & 9 & 5131 & 16.45 & 48 & 14 & 28 \\
\hline R DLPFC—-middle frontal gyrus & 46 & & 13.63 & 50 & 32 & 18 \\
\hline $\mathrm{R}$ Inferior frontal gyrus & 47 & & 9.73 & 34 & 26 & 0 \\
\hline R Posterior cerebellum—crus I, 2 & & 146 & 9.14 & 12 & -82 & -30 \\
\hline R Inferior parietal lobe & 40 & 1448 & 8.48 & 36 & -56 & 46 \\
\hline R Supramarginal gyrus & 40 & & 8.16 & 48 & -44 & 36 \\
\hline R Inferior parietal lobe & 40 & & 8.06 & 52 & -44 & 46 \\
\hline R Posterior cerebellum - crus 2 & & 407 & 7.39 & 36 & -74 & -46 \\
\hline $\mathrm{R}$-crus 2 & & & 7.33 & 30 & -80 & -44 \\
\hline$R$-crus I & & & 6.97 & 34 & -68 & -30 \\
\hline
\end{tabular}

Abbreviations: ACC, anterior cingulate cortex; ALC, alcoholic; CTL, control; IDLPFC, left dorsolateral prefrontal cortex.

Thresholds for group effects and interactions were set at $P_{\text {FDR-corrected }}<0.05$ for the whole brain (combined height and extent threshold; Poline et al, 1997); for group similarities, the peak threshold was set at $P_{\text {FWE-corrected }}<0.05$ for the whole brain.
Left DLPFC seed. In general, ALC and CTL showed overlapping functional connectivity to lateral frontoparietal regions of both hemispheres and to right cerebellar Crus1 and Crus2. Compared with CTL, ALC showed less synchronous activity with left anterior cingulate and inferior frontal cortices, and a left anterior cerebellum cluster (region 4,5) extending into the right Crus1. At the same time, ALC showed more synchronous activity to a left insula cluster that extended into middle frontal and medial temporal gyri (Figure 2b, Table 4).

\section{Correlation between ROI Activity, Independent Emotion Measures, and Craving Scores}

Higher trait anxiety scores correlated with greater midbrain and parahippocampal activity during Stroop conditions involving alcohol (midbrain: $r=0.56, P_{\mathrm{FDR} \text {-corrected }}=0.002$; parahipp: $\left.r=0.48, P_{\text {FDR-corrected }}=0.007\right)$, negative emotion (midbrain: $r=0.65, P_{\text {FDR-corrected }}<0.0001$; parahipp: $r=0.60$, $P_{\text {FDR-corrected }}=0.001$ ), and positive emotion (midbrain: $r=0.55, P_{\mathrm{FDR} \text {-corrected }}=0.002$; parahipp: $r=0.52, P=0.004$ ). Higher trait anxiety scores in ALC further correlated with lower left DLPFC activity during positive-emotion Stroop (lDLPFC: $\left.r=-0.51, P_{\text {FDR-corrected }}=0.005\right)$ and alcohol Stroop (lDLPFC: $r=-0.38, P_{\text {FDR-corrected }}=0.03$ ). There were no significant relations between ROI activity and depression and anxiety scores in control subjects (all $P$ 's $>0.05$ ). Lower midbrain activity during emotionally neutral color Stroop in ALC correlated with higher depressive symptoms (BDI-II; $\left.r=-0.46, P_{\text {uncorrected }}=0.012\right)$ and trait anxiety (STAI-T; $\left.r=-0.37, P_{\mathrm{FDR} \text {-corrected }}=0.034\right)$ scores. Higher alcohol craving scores in ALC correlated with greater behavioral alcohol Stroop effects $\left(r=0.50, P_{\text {FDR- }}\right.$ corrected $=0.007$ ), higher parahippocampal activity during alcohol Stroop processing $\left(r=0.39, P_{\text {uncorrected }}=0.025\right)$, and lower IDLPFC activity during positive-emotion Stroop processing $\left(r=-0.45, P_{\mathrm{FDR}-\text { corrected }}=0.011\right)$, but not in CTL (all $P>0.05$ ).

\section{DISCUSSION}

Our study provides evidence that chronic ALC differ from CTL in activation of frontoparietal executive control and midbrain-based reward networks. Priming these functions by using a novel alcohol-emotion Stroop paradigm, we found interference from both incongruent color words and from alcohol words. Behavioral Stroop effects, however, were not forthcoming for emotional words. ALC had, as a trend, greater Stroop effects for color words than CTL, and showed significantly greater color Stroop effects for response-repetition conditions. Similar to previous findings (Schulte et al, 2012), and in contrast to CTL, ALC did not profit from repetition priming when resolving Stroop color-word conflict.

Our functional neuroimaging data revealed the expected frontoparietal activation pattern during color Stroop processing in both groups. Most importantly, group differences emerged for midbrain activation patterns when comparing alcohol Stroop to color Stroop processing, and for left DLPFC activity when comparing positive-emotion Stroop with color Stroop processing. Here, ALC showed 
more midbrain activation during alcohol-related word Stroop and less DLPFC activation during positive-emotion word processing than CTL. Although repeated exposure to alcohol leads to a decrease in dopamine release in the nucleus accumbens and striatum (Martinez et al, 2005; Volkow et al, 2007), we speculate that the enhanced midbrain/parahippocampal activation to alcohol-related words suggests neuroadaptations of dopaminergic midbrain systems (Bowirrat and Oscar-Berman, 2005; Perra et al, 2011). Such tuning is normally associated with behavioral conditioning to optimize responses, but here it contributed to automatic bias to alcohol-related stimuli. This is consistent with a recent meta-analysis of fMRI studies on alcohol cue reactivity indicating engagement of ventral striatal and prefrontal regions to alcohol cue exposure (Schacht et al, 2013). These neural patterns may have a key role in the emotional misbalance and feelings of anxiety that can drive alcohol use and serve as risk factors for relapse (Koob and Volkow, 2010).

Midbrain responsiveness to emotion and alcohol-related word challenge, in contrast to cognitive challenge, may characterize the individual's degree of neural dysregulation and vulnerability for emotional disturbance and alcohol addiction. Dampened prefrontal activations have been previously associated with poor response inhibition in the development of alcohol dependence (Courtney et al, 2012). Recent imaging studies provided convergent evidence by showing less anterior cingulate activation during simple decoding of negative emotional facial expressions in ALC than CTL (Salloum et al, 2007). The low prefrontal activation to positive emotional content in the ALC relative to CTL in our study adds to these findings and suggests downregulation of the frontal monitoring system responsiveness, even for positive-emotion. In addition, current smoking was associated with lower activation in this downregulation, demonstrating the adverse interactive effects of alcoholism and smoking on prefrontal responsiveness to positiveemotion. Thus, substantial diagnosis-specific differences occurred in the neural midbrain-frontal substrates for emotional and alcohol-related word processing.

Brain activity to alcohol-related word and emotionspecific word challenge in ALC correlated with independent questionnaire-based emotion measures. ALC with higher trait anxiety scores activated midbrain and parahippocampal regions more during alcohol-related word and emotion Stroop processing, but not during color Stroop processing, than ALC with lower trait anxiety scores. Higher trait anxiety in ALC was further associated with lower left DLPFC activity during positive-emotion Stroop and alcohol-related word Stroop processing. The opposite relationship was observed for the relative low midbrain activity in ALC during emotionally neutral color Stroop processing. Midbrain activity correlated with higher depressive symptoms and trait anxiety scores, possibly reflecting an altered balance in chronic ALC within a complex midbrainfrontostriatal adaptation network involved in emotion and reward regulation (Colibazzi et al, 2010; Golkar et al, 2012). In addition, higher alcohol craving in abstinent ALC correlated with higher parahippocampal activity during alcohol-Stroop and lower DLPFC activity during positive-emotion processing. Consistent with previous findings (Heinz et al, 2007, 2010), such alcohol- and emotion-specific activation patterns may pertain to relapse risk prediction.

Together, our findings demonstrated diagnosis-specific differences with lower DLPFC activation indicating reduced neural response to processing positive-emotional content, and greater midbrain and parahippocampal activation in ALC than CTL when processing alcohol-related words relative to color words. Here, a significant overlap of the midbrain activation was observed for alcohol-related and negative-emotion processing in ALC. Thus, in ALC the mesolimbic corticostriatal adaptive neural 'alarm' system for rapid alerting (Liddell et al, 2005) is not only responding more to sources of high emotional relevance but also to alcohol-related stimuli. This is consistent with the hypothesis that alcohol-related stimuli gain emotional relevance with continued alcohol use. In addition, compared with CTL, functional midbrain connectivity in ALC was lower in prefrontal cortices involved in emotion regulation and was more to caudate, thalamus, and cerebellar regions involved in valence and arousal dimensions of emotions, appetitive experiences, and cue-induced craving. This pattern provides evidence for an alcohol cue-induced deregulation of midbrain-based mesolimbic corticostriatal circuitry for reward and emotion regulation in abstinent ALC and may delineate the neural underpinnings for the risk of relapse when confronted with individual relevant alcohol cues or negative emotions.

\section{FUNDING AND DISCLOSURE}

The authors declare no conflict of interest.

\section{ACKNOWLEDGEMENTS}

We thank Stephanie Sassoon, Priya Asok, Karen Jackson, and Crystal Caldwell for help with recruitment and clinical interviewing, and Fiona Baker for comments on the manuscript. NIH Grants R01 AA018022, AA012388, and AA017168 funded this work.

\section{REFERENCES}

American Psychiatric Association (2000). Diagnostic and Statistical Manual of Mental Disorders. 4th ed. American Psychiatric Press: Washington, DC.

Beck AT, Steer RA, Brown GK (1996). Manual for the Beck Depression Inventory-II. Psychological Corporation: San Antonio, TX.

Benjamini Y, Drai D, Elmer G, Kafkafi N, Golani I (2001). Controlling the false discovery rate in behavior genetics research. Beh Brain Res 125: 279-284.

Beresford TP, Arciniegas DB, Alfers J, Clapp L, Martin B, Du Y et al (2006). Hippocampus volume loss due to chronic heavy drinking. Alcohol Clin Exp Res 30: 1866-1870.

Berridge KC, Robinson TE (2003). Parsing reward. Trends Neurosci 26: $507-513$.

Bowirrat A, Oscar-Berman M (2005). Relationship between dopaminergic neurotransmission, alcoholism, and Reward Deficiency syndrome. Am J Med Genet B Neuropsychiatr Genet 132B: 29-37, Review.

Bush G, Luu P, Posner MI (2000). Cognitive and emotional influences in anterior cingulate cortex. Trends Cogn Sci 4: $215-222$. 
Clark US, Oscar-Berman M, Shagrin B, Pencina M. (2007). Alcoholism and judgments of affective stimuli. Neuropsychology 21: $346-362$.

Cohen JD, MacWhinney B, Flatt M, Provost J (1993). PsyScope: A new graphic interactive environment for designing psychology experiments. Beh Res Methods Instrum Comput 25: 257-271.

Colibazzi T, Posner J, Wang Z, Gorman D, Gerber A, Yu S et al (2010). Neural systems subserving valence and arousal during the experience of induced emotions. Emotion 10: 377-389.

Courtney KE, Ghahremani DG, Ray LA (2012). Fronto-striatal functional connectivity during response inhibition in alcohol dependence. Addict Biol 18: 593-604.

De Rosa E, Desmond JE, Anderson AK, Pfefferbaum A, Sullivan EV (2004). The human basal forebrain integrates the old and the new. Neuron 41: 825-837.

Devinsky O, Morrell MJ, Vogt BA (1995). Contributions of anterior cingulate cortex to behaviour. Brain 118: 279-306.

Di Chiara G (2002). Nucleus accumbens shell and core dopamine: differential role in behavior and addiction. Behav Brain Res 137: 75-114.

Evert DL, Oscar-Berman M (2001). Selective attentional processing and the right hemisphere: effects of aging and alcoholism. Neuropsychology 15: 452-461.

Fein G, Chang M (2006). Visual P300s in long-term abstinent chronic alcoholics. Alcohol Clin Exp Res 30: 2000-2007.

Field M, Mogg K, Mann B, Bennett GA, Bradley BP (2012). Attentional Biases in Abstinent Alcoholics and Their Association With Craving. Psychol Addict Behav 27: 71-80.

Fitzgerald DA, Angstadt M, Jelsone LM, Nathan PJ, Phan KL (2006). Beyond threat: amygdala reactivity across multiple expressions of facial affect. Neuroimage 30: 1441-1448.

Foisy ML, Kornreich C, Fobe A, D’Hondt L, Pelc I, Hanak C et al (2007). Impaired emotional facial expression recognition in alcohol dependence: do these deficits persist with midterm abstinence? Alcohol Clin Exp Res 31: 404-410.

Friston KJ, Frith CD, Frackowiak RS, Turner R (1995). Characterizing dynamic brain responses with fMRI: a multivariate approach. Neuroimage 2: 166-172.

Friston KJ, Penny WD, Glaser DE (2005). Conjunction revisited. Neuroimage 25: 661-667.

Gainotti G (2001). Disorders of emotional behaviour. J Neurol 248: 743-749.

Gilman JM, Hommer DW (2008). Modulation of brain response to emotional images by alcohol cues in alcohol-dependent patients. Addict Biol 13: 423-434.

Gilman JM, Ramchandani VA, Davis MB, Bjork JM, Hommer DW (2008). Why we like to drink: a functional magnetic resonance imaging study of the rewarding and anxiolytic effects of alcohol. I Neurosci 28: 4583-4591.

Glahn DC, Lovallo WR, Fox PT (2007). Reduced amygdala activation in young adults at high risk of alcoholism: studies from the Oklahoma Family Health Patterns Project. Biol Psychiatry 61: 1306-1309.

Golkar A, Lonsdorf TB, Olsson A, Lindstrom KM, Berrebi J, Fransson $\mathrm{P}$ et al (2012). Distinct contributions of the dorsolateral prefrontal and orbitofrontal cortex during emotion regulation. PLoS One 7: e48107.

Grüsser SM, Wrase J, Klein S, Hermann D, Smolka MN, Ruf M et al (2004). Cue-induced activation of the striatum and medial prefrontal cortex is associated with subsequent relapse in abstinent alcoholics. Psychopharmacology (Berl) 175: 296-302.

Heinz A, Wrase J, Kahnt T, Beck A, Bromand Z, Grüsser SM et al (2007). Brain activation elicited by affectively positive stimuli is associated with a lower risk of relapse indetoxified alcoholic subjects. Alcohol Clin Exp Res 31: 1138-1147.

Heinz A, Beck A, Mir J, Grüsser SM, Grace AA, Wrase J (2010). Alcohol craving and relapse prediction: imaging studies In: Kuhn CM, Koob GF (eds) Advances in the Neuroscience of
Addiction. 2nd edn. Chapter 4. Frontiers in Neuroscience. CRC Press: Boca Raton (FL).

Johnsen BH, Laberg JC, Cox WM, Vaksdal A, Hugdahl K (1994). Alcoholic subjects' attentional bias in the processing of alcoholrelated words. Psychol Addict Behav 8: 111-115.

Koob GF, Volkow ND (2010). Neurocircuitry of addiction. Neuropsychopharmacology 35: 217-238.

Le Berre AP, Rauchs G, La Joie R, Mézenge F, Boudehent C, Vabret $\mathrm{F}$ et al (2012). Impaired decision-making and brain shrinkage in alcoholism. Eur Psychiatry [Epub ahead of print].

Liddell BJ, Brown KJ, Kemp AH, Barton MJ, Das P, Peduto A et al (2005). A direct brainstem-amygdala-cortical 'alarm' system for subliminal signals of fear. Neuroimage 24: 235-243.

Makris N, Oscar-Berman M, Jaffin SK, Hodge SM, Kennedy DN, Caviness VS et al (2008). Decreased volume of the brain reward system in alcoholism. Biol Psychiatry 64: 192-202.

Mann K, Agartz I, Harper C, Shoaf S, Rawlings RR, Momenan R et al (2001). Neuroimaging in alcoholism: ethanol and brain damage. Alcohol Clin Exp Res 25, (5 Suppl ISBRA) 104S-109S.

Marinkovic K, Oscar-Berman M, Urban T, O’Reilly CE, Howard JA, Sawyer K et al (2009). Alcoholism and dampened temporal limbic activation to emotional faces. Alcohol Clin Exp Res 33: 1880-1892.

Martinez D, Gil R, Slifstein M, Hwang DR, Huang Y, Perez A et al (2005). Alcohol dependence is associated with blunted dopamine transmission in the ventral striatum. Biol Psychiatry 58: 779-786.

Maurage P, Grynberg D, Noël X, Joassin F, Hanak C, Verbanck P et al (2011). The 'Reading the Mind in the Eyes' test as a new way to explore complex emotions decoding in alcohol dependence. Psychiatry Res 190: 375-378.

Montagne B, Kessels RP, Wester AJ, de Haan EH (2006). Processing of emotional facial expressions in Korsakoffs syndrome. Cortex 42: 705-710.

Morrison SE, Salzman CD (2009). The convergence of information about rewarding and aversive stimuli in single neurons. I Neurosci 29: 11471-11483.

Müller-Oehring EM, Schulte T, Fama R, Pfefferbaum A, Sullivan EV (2009). Global-local interference is related to callosal compromise in alcoholism: a behavior-DTI association study. Alcohol Clin Exp Res 33: 477-489.

Nichols T, Brett M, Andersson J, Wager T, Poline JB (2005). Valid conjunction inference with the minimum statistic. Neuroimage 25: 653-660.

Oberlin BG, Dzemidzic M, Bragulat V, Lehigh CA, Talavage T, O'Connor SJ et al (2012). Limbic responses to reward cues correlate with antisocial trait density in heavy drinkers. Neuroimage 60: 644-652 s.

O’Daly OG, Trick L, Scaife J, Marshall J, Ball D, Phillips ML et al (2012; Withdrawal-associated increases and decreases in functional neural connectivity associated with altered emotional regulation in alcoholism. Neuropsychopharmacology 37: 2267-2276.

Oscar-Berman M, Hancock M, Mildworf B, Hutner N, Weber DA (1990). Emotional perception and memory in alcoholism and aging. Alcohol Clin Exp Res 14: 383-393.

Oscar-Berman M (2000). Neuropsychological vulnerabilities in chronic alcoholism. In: Noronha A, Eckardt MJ. Warren K (eds). Review of NIAAA's Neuroscience and Behavioral Research Portfolio Vol. Monograph No. 34 (Department of Health and Human Services: Bethesda, MD, USA, pp 437-471.

Oscar-Berman M, Bowirrat A (2005). Genetic influences in emotional dysfunction and alcoholism-related brain damage. Neuropsychiatr Dis Treat 1: 211-229.

Perra S, Clements MA, Bernier BE, Morikawa H (2011). In vivo ethanol experience increases $\mathrm{D}(2)$ autoinhibition in the ventral tegmental area. Neuropsychopharmacology 36: 993-1002.

Phillips SC, Harper CG, Kril J (1987). A quantitative histological study of the cerebellar vermis in alcoholic patients. Brain 110: 301-314. 
Phillips ML, Ladouceur CD, Drevets WC (2008). A neural model of voluntary and automatic emotion regulation: implications for understanding the pathophysiology and neurodevelopment of bipolar disorder. Mol Psychiatry 13: 833-857.

Pfefferbaum A, Sullivan EV, Mathalon DH, Lim KO (1997). Frontal lobe volume loss observed with magnetic resonance imaging in older chronic alcoholics. Alcohol Clin Exp Res 21: 521-529.

Poline JB, Worsley KJ, Evans AC, Friston KJ (1997). Combining spatial extent and peak intensity to test for activations in functional imaging. Neuroimage 5: 83-96.

Salloum JB, Ramchandani VA, Bodurka J, Rawlings R, Momenan $\mathrm{R}$, George $\mathrm{D}$ et al (2007). Blunted rostral anterior cingulate response during a simplified decoding task of negative emotional facial expressions in alcoholic patients. Alcohol Clin Exp Res 31: 1490-1504.

Schacht JP, Anton RF, Myrick H (2013). Functional neuroimaging studies of alcohol cue reactivity: a quantitative meta-analysis and systematic review. Addict Biol 18: 121-133.

Schneider F, Habel U, Wagner M, Franke P, Salloum JB, Shah NJ et al (2001). Subcortical correlates of craving in recently abstinent alcoholic patients. Am J Psychiatry 158: 1075-1083.

Schulte T, Mueller-Oehring EM, Rosenbloom MJ, Pfefferbaum A, Sullivan EV (2005). Differential effect of HIV infection and alcoholism on conflict processing, attentional allocation, and perceptual load: evidence from a Stroop Match-to-Sample task. Biol Psychiatry 57: 67-75.

Schulte T, Müller-Oehring EM, Javitz H, Pfefferbaum A, Sullivan EV (2008). Callosal compromise differentially affects conflict processing and attentional allocation in alcoholism, HIV, and their comorbidity. Brain Imaging Behav 2: 27-38.

Schulte T, Müller-Oehring EM, Pfefferbaum A, Sullivan EV (2010). Neurocircuitry of emotion and cognition in alcoholism: contributions from white matter fiber tractography. Dialogues Clin Neurosci 12: 554-560.

Schulte T, Müller-Oehring EM, Sullivan EV, Pfefferbaum A (2011). Disruption of emotion and conflict processing in HIV infection with and without alcoholism comorbidity. J Int Neuropsychol Soc 22: $1-14$

Schulte T, Müller-Oehring EM, Sullivan EV, Pfefferbaum A (2012). Synchrony of corticostriatal-midbrain activation enables normal inhibitory control and conflict processing in recovering alcoholic men. Biol Psychiatry 71: 269-278.

Schulte T, Müller-Oehring EM, Vinco S, Hoeft F, Pfefferbaum A, Sullivan EV (2009). Double dissociation between actiondriven and perception-driven conflict resolution invoking anterior versus posterior brain systems. Neuroimage 48: 381-390.

Sinha R, Li CS (2007). Imaging stress- and cue-induced drug and alcohol craving: association with relapse and clinical implications. Drug Alcohol Rev 26: 25-31.

Spielberger CD, Gorsuch RL, Lushene R, Vagg PR, Jacobs GA (1983). Manual for the State-Trait Anxiety Inventory. Consulting Psychologists Press: Palo Alto, CA.

Sullivan EV, Rosenbloom MJ, Lim KO, Pfefferbaum A (2000). Longitudinal changes in cognition, gait, and balance in abstinent and relapsed alcoholic men: relationships to changes in brain structure. Neuropsychology 14: 178-188.

Sullivan EV, Harding AJ, Pentney R, Dlugos C, Martin PR, Parks MH et al (2003). Disruption of frontocerebellar circuitry and function in alcoholism. Alcohol Clin Exp Res 27: 301-309.

Sullivan EV, Deshmukh A, De Rosa E, Rosenbloom MJ, Pfefferbaum A (2005). Striatal and forebrain nuclei volumes: contribution to motor function and working memory deficits in alcoholism. Biol Psychiatry 57: 768-776.

Townshend JM, Duka T (2003). Mixed emotions: alcoholics' impairments in the recognition of specific emotional facial expressions. Neuropsychologia 41: 773-782.

Volkow ND, Wang GJ, Telang F, Fowler JS, Logan J, Jayne M et al (2007). Profound decreases in dopamine release in striatum in detoxified alcoholics: possible orbitofrontal involvement. J Neurosci 27: 12700-12706.

Vollstädt-Klein S, Loeber S, Richter A, Kirsch M, Bach P, von der Goltz C et al (2012). Validating incentive salience with functional magnetic resonance imaging: association between mesolimbic cue reactivity and attentional bias in alcohol-dependent patients. Addict Biol 17: 807-816.

Wrase J, Makris N, Braus DF, Mann K, Smolka MN, Kennedy DN et al (2008). Amygdala volume associated with alcohol abuse relapse and craving. Am J Psychiatry 165: 1179-1184.

Supplementary Information accompanies the paper on the Neuropsychopharmacology website (http://www.nature.com/npp) 